\title{
Lifecycle Health Assessment Model for Sustainable Healthy Buildings
}

\author{
Lee, Sungho Lim, Chaeyeon Kim, Sunkuk* \\ Department of Architectural Engineering, Kyung Hee University, Giheung-Gu, Yongin, 446-701, Korea
}

\begin{abstract}
A system to analyze, assess and manage the health performance of resources and spaces throughout the project lifecycle shall be established to ensure sustainable healthy buildings. Decisions made in the planning, design, construction, and operation and management (O\&M) phases must help sustain the health performance of buildings at the level specified by clients or the relevant laws. For this reason, it is necessary to develop a model to ensure the consistent management of performance, as such performance varies according to the decisions made by project participants in each phase. The purpose of this research is to develop a Lifecycle Health Assessment Model (LHA) for sustainable healthy buildings. The developed model consists of four different modules: the Health-friendly Resources Database (HRDB) module, which provides health performance data regarding resources and spatial elements; the Lifecycle Health-performance Tree (LHT) module, which analyzes the hierarchy of spatial and health impact factors; the Health Performance Evaluation (HPE) Module; and the Lifecycle Health Management Module, which analyzes and manages changes in health performances throughout the lifecycle. The model helps ensure sustainable health performances of buildings.
\end{abstract}

Keywords : lifecycle, health assessment, health management, health performance tree, health-friendly resources

\section{Introduction}

It is necessary to establish a system that enables the easy and rapid evaluation of the health performance data used for sustainable healthy buildings $[1,2,3]$, and the analysis, assessment, and efficient management of the health performance of buildings throughout their lifecycles[4,5,6,7]. With this aim, in previous research, a Health-friendly Resources Database (HRDB) [8] was developed that can integrate resources as basic data for the comprehensive assessment of the health performance of whole buildings, along with a Lifecycle Health-performance Tree (LHT) model[9] that evaluates health

\section{Received : February 7, 2014}

Revision received : March 6, 2014

Accepted : June 25, 2014

* Corresponding author: Kim, Sunkuk

[Tel: 82-31-201-2922, E-mail: kimskuk@khu.ac.kr]

(c)2014 The Korea Institute of Building Construction, All rights reserved. performance. A computing model for systematic evaluation of the health performance of buildings was then developed by adding process and data models to the LHT model to develop the HPE model[3]. As the models developed through previous studies focus on the assessment and evaluation of a building's health performance, models to integrate, share, and manage information in accordance with lifecycles have yet to be developed.

To assure comprehensive management, the evaluation of the health performance of buildings in each lifecycle phase is necessary. In each phase, decisions will be made to maintain a specific level of health performance. The health performance of buildings varies according to the decisions made by project participants in respective phases. Therefore, it is necessary to develop a model that enables the management of the health performance.

The purpose of this study is to develop a Lifecycle Health Assessment (LHA) model for sustainable 
healthy buildings. The LHA model consists of four different modules: the Health-friendly Resources Database (HRDB) module, which provides health performance data regarding resources and Space Configuration Items (SCIs); the Lifecycle Health-performance Tree (LHT) module, which analyzes the hierarchy of spaces and health impact factors; the Health Performance Evaluation (HPE) module, which evaluates the health performance of a space; and the Lifecycle Health Management module, which analyzes and manages change in health performance throughout the lifecycle. The model helps ensure the sustainable health performance of buildings.

The research process is as follows: First, HRDB, LHT, and HPE models are reviewed. Second, module configuration of LHA and concepts of the Lifecycle Health Management (LHM) module are explained. Third, the concepts and operating methodologies of the LHA model suggested in this study are proposed.

\section{Preliminary Research}

The LHA model developed in this study provides structures, data, and methodologies for evaluating the health performance of buildings and sharing the results conducted throughout the lifecycles, as shown in Figure 1. Among the modules specified herein, HRDB, LHT, and HPE were already developed[3,8,9]. The relationships between those modules consist of LHT determining the hierarchy of spaces and health impact elements, the information exchange of HRDB offering health performance information regarding resources and SCIs in accordance with such structure, and HPE. Finally, the Lifecycle Health Management (LHM) module is used to analyze and control changes in health performance throughout lifecycles. The LHA model is designed as a system to enable the comprehensive management of these modules. This chapter reviews HRDB, LHT, and HPE modules, which were developed through previous studies.

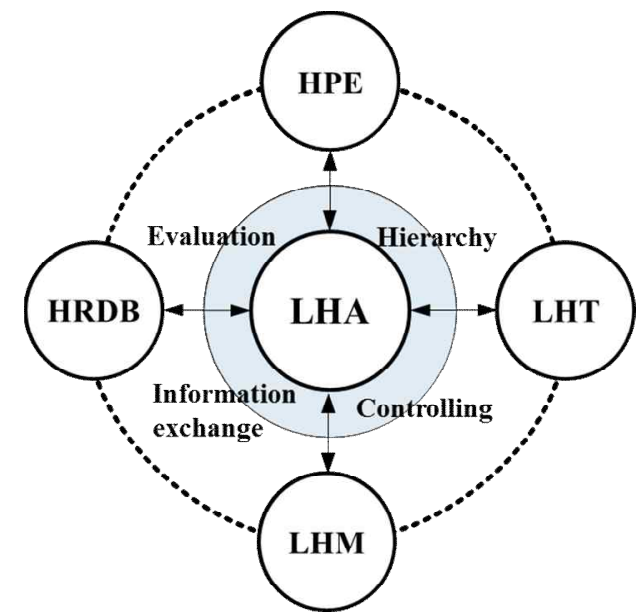

Figure 1. LHA model concept

\subsection{HRDB}

HRDB refers to the establishment of a database for the analysis of health performance of resources supplied in the construction market[8]. The categories of healthy building information were analyzed while the formalities of data collection were designed in association with health performance indices in order to establish HRDB. A data-associated operating algorithm was then set up using the HRDB structure design and an entity-relation diagram. HRDB was designed to enable the rapid search and sharing of health-friendly resource information in the planning and design phases in order to expedite decision-making processes[14]. It helps assure fast updates and upgrades of information produced in the construction and operation phases, during which selected materials are used.

Figure 2 shows the HRDB management concept. The manufacturing company produces the appropriate materials and services by searching HRDB for the legal and performance criteria. The company then examines the materials and services, and requests the authorized organizations for certification. In ad- 
dition, the major stockholders use the materials information from the management system in each phase, and input the health performance information of installed materials and services[8].

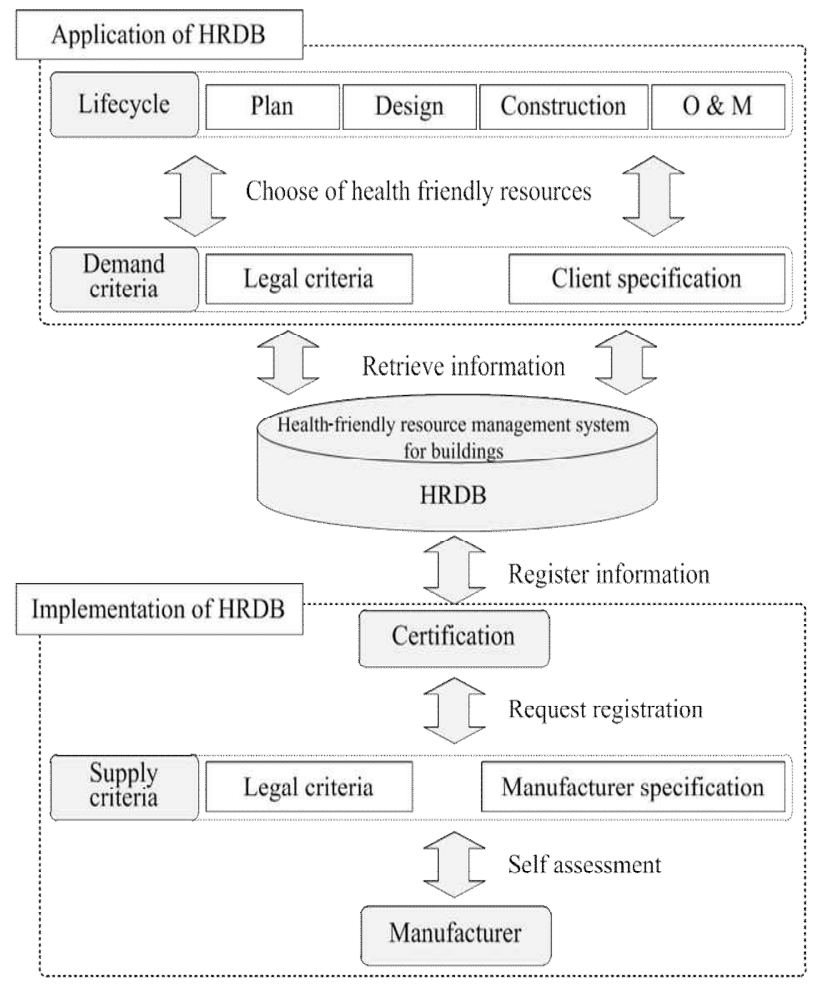

Figure 2. HRDB application concept[8]

\subsection{LHT}

LHT is a technology that determines the relationships between factors that affect the health of residents and SCIs to assess spatial health performance after categorizing such factors into light, sound, heat, and air, as shown in Figure 3. LHT is a tool to provide a health performance evaluation system in consideration of the lifecycle based on the unit space of a building. LHT helps categorize SCIs into air, heat, sound, and light in accordance with the physical impacts on residents by using concepts that integrate the lifecycle into space analysis. Those health performance factors enable the separation of a variety of health performances of SCIs. SCIs such as opening, space, and various facilities are difficult to convert directly into health performance, so LHT quantifies health performance through the categorized analysis. LHT not only targets materials and spaces, but also extends its evaluation coverage to entire buildings and project lifecycle phases[9].

In other words, LHT provides information that aids in the evaluation of the health performance of SCIs by space using evaluation factors (EFs) and health performance factors (HFs), as shown in Figure 3. HF indicates the main categories such as indoor air quality, heating, and cooling, heating, ventilation, and air conditioning (HVAC) and lighting, while EF refers to the sub-categories. For example, IAQ can be affected by gases (including carbon monoxide, radon, and volatile organic compounds), particulates, microbial contaminants (mold, bacteria) or any mass or energy stressor that can induce adverse health conditions $[10,12,13]$.

\subsection{HPE}

$\mathrm{HPE}$ is the concept in which the health performance of a building is evaluated and data production is shared throughout the lifecycle of a healthy building. Figure 4 indicates the overall concepts of HPE, consisting of three different axes (target, indicator, and time). This suggests that HPE evaluates the health performance of spaces and resources, and shares information throughout the lifecycle of a building quickly and easily[3].

The configuration of HPE is associated with process models, information models, and LHT modules. The process model is designed to assure the easy and quick evaluation of health performance through the management of operating procedures. The information model is designed to control the health performance information of resources and to store and retrieve necessary information in association with HRDB[3]. 
Spaces Space Configuration Items (SCIs) $\quad \frac{\text { Health performance }}{\underline{\text { Factors (HFs) }}} \quad$ Evaluation results

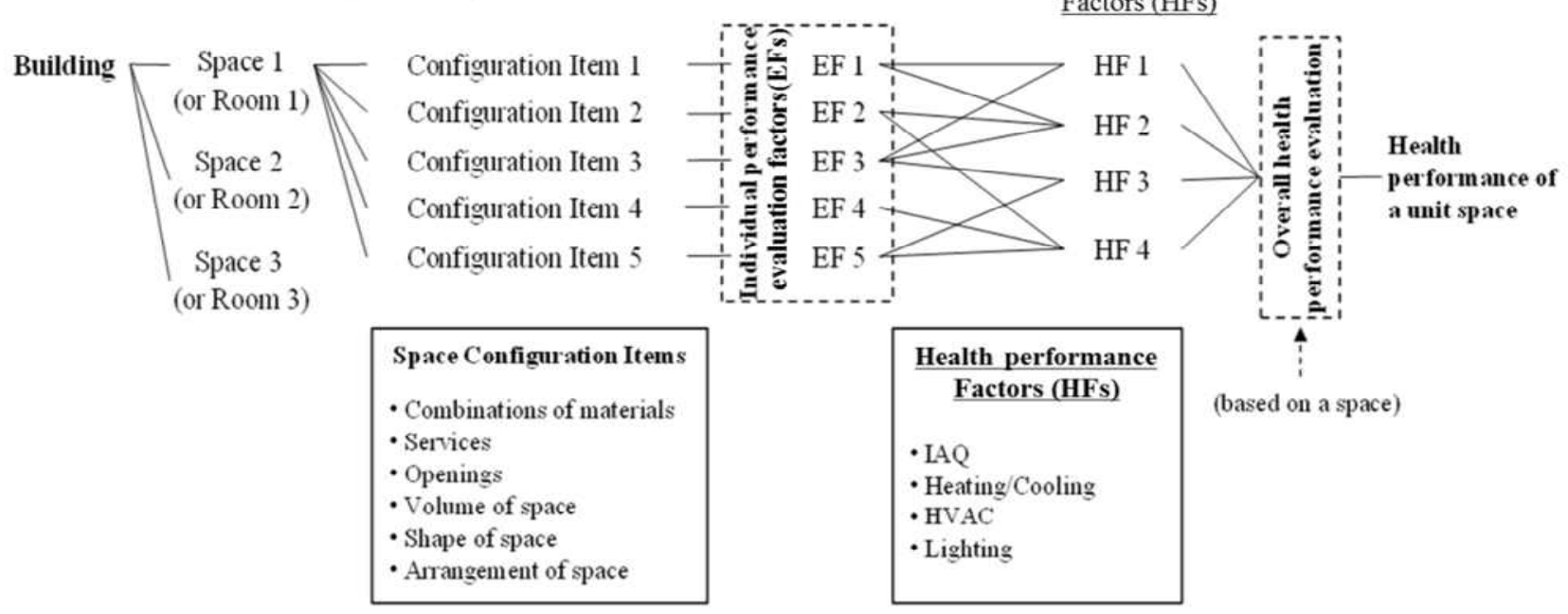

Figure 3. Configuration factors of the space and health performance[9]

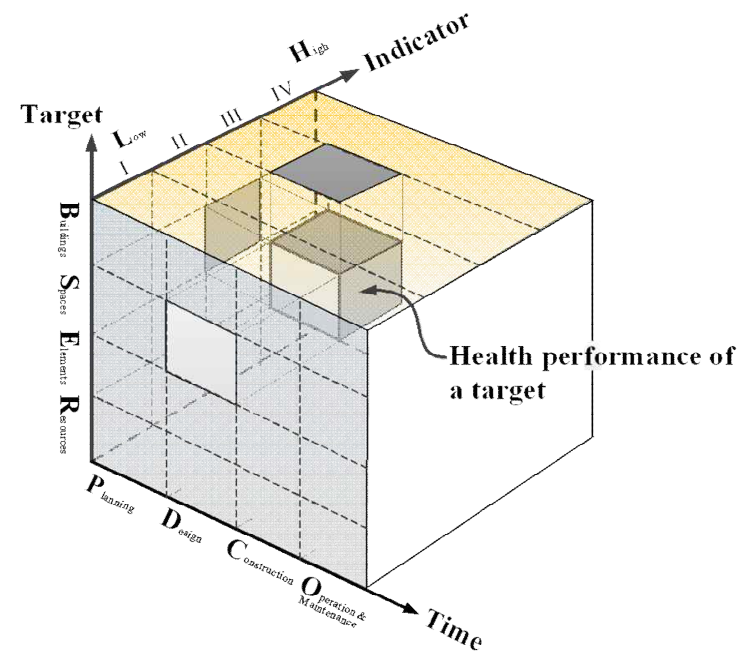

Figure 4. HPE model concept[3]

\section{LHA Model}

\subsection{Module configuration of the LHA model}

The LHA model consists of LHT, HRDB, HPE, and LHM modules to analyze, assess, and control the health performance of buildings throughout their lifecycles, as shown in Figure 5[3,8,9]. The LHT module is designed to control spaces, SCIs, EFs, and HFs systematically, as described above[9], while the HRDB module is designed to provide the properties, health performance and cost of health-related mate- rials and services[8]. In addition, the HPE module quantifies, classifies, regularizes and evaluates the health performance of spaces and resources (materials and services)[3]. Meanwhile, the LHM module is designed to measure health performance, to provide a comparative analysis of the pre-process and post-process phases, and to provide a baseline for health performance.

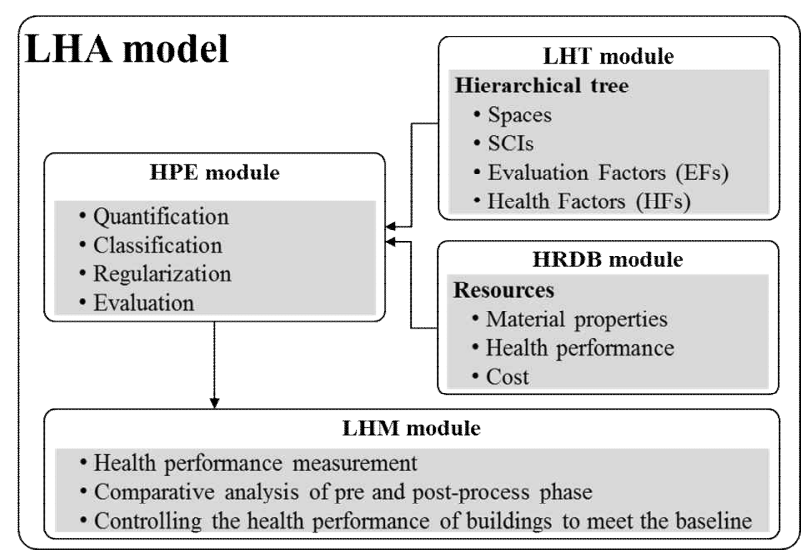

Figure 5. Module configuration of the LHA model

For reference, LHA is used as a tool to measure a building' s health performance and analyze the corresponding variations in the planning, design, construction, and operation and management phases to 
ensure sustainable healthy buildings. In contrast, life cycle assessment (LCA) measures the total $\mathrm{CO}_{2}$ production of the entire lifecycle.

LHA measures the health performance of a building at a specific time. For example, LHA can confirm the health performance of a building when the design process is complete. Figure 6 visualizes the relationship between the building lifecycle and LHA. The health performance of a building at a specific time is composed of the LHT, combining space, space configuration items (SCIs), indicators and HPE evaluating health performance based on HRDB, which consists of material properties, health performance, and costs. The performance measurements and information produced repetitively in the planning, design, construction, and operation and management phases are continuously managed by LHM until the project is complete, and thus are used as basic data input to maintain the proper level of health performance for the building. For instance, the analysis of health performance using LHA in the design phase can be stipulated as shown in Figure 7. HRBD, LHT, and HPE models are integrated in accordance with the building lifecycle to analyze the health performance of each building.

At this point, the LHM module maintains the minimum limitation of health performance (the so-called baseline of health performance) to ensure comfortable living environments for residents throughout the building lifecycle. The health performance must be assessed at a specific time in order to confirm that it is properly maintained; this is referred to as the pre-process and post-process phase. This means that LHM is a procedure for measuring the building' $\mathrm{s}$ health performance and analyzing the identified changes for assessment. Since the health performance is maintained in the pre-process phase, but fails to meet the baseline in the post-process phase, efforts must be made to maintain the building' $\mathrm{s}$ health performance. Under LHM, investments and other efforts must be made to upgrade health performance when it is measured as below the baseline of health performance. A proper health performance level must be assured in accordance with the purpose of use throughout the building lifecycle; such efforts are undertaken by LHM.

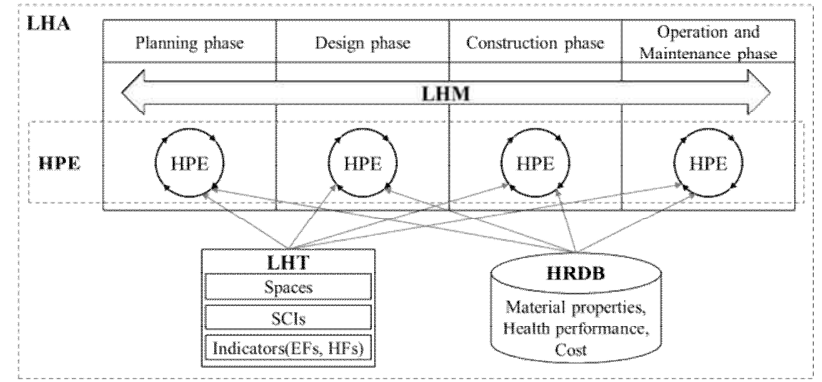

Figure 6. Relation of building lifecycle and LHA

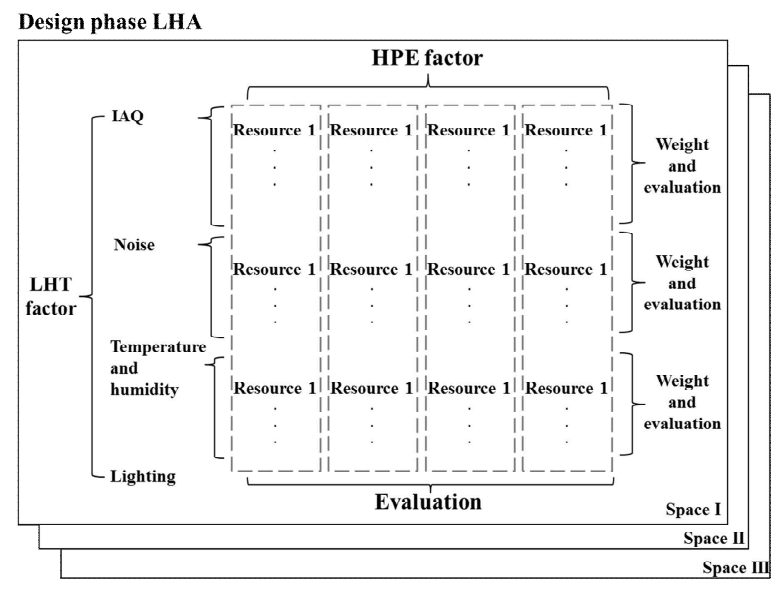

Figure 7. LHA detail model

\subsection{Application process of the LHA model}

It is necessary to apply different evaluation methodologies to the comprehensive health performance of a building in each lifecycle phase. Visualizing the operating procedures as shown in Figure 8, the health performance plan must be established on the basis of legal standards and the health performance requirements of the client in Figure 9. HPE technology established in the planning phase is then utilized to assess health performance. Based on the assessment, a building design is produced. Eq. (1) is used 


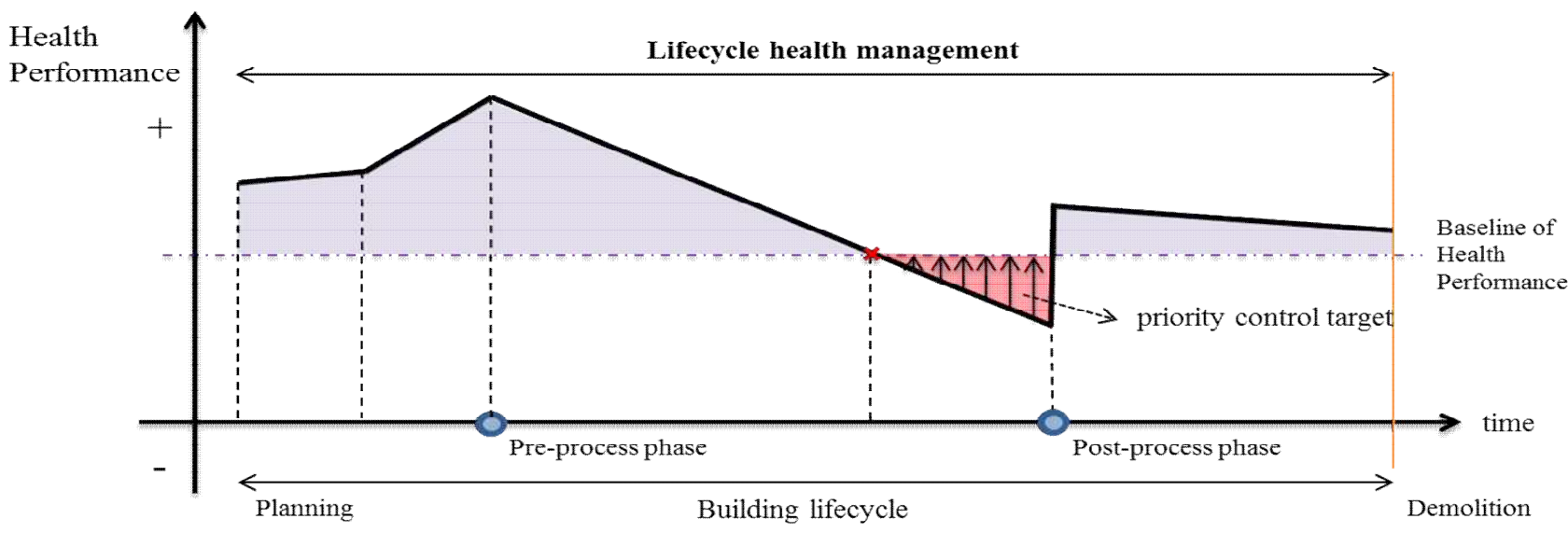

Figure 8. Lifecycle health management concept

to assess the health performance of a building using HPE on the basis of LHT and HRDB[3]. The health performance assessment is examined to determine whether it meets functional requirements, as indicated in Eq. (2). The functional requirement refers to the baseline specified in Figure 8, which is determined according to the requirements of clients or legal criteria. The client's requirement is to have a higher building performance and health performance than the legal criteria.

$$
\begin{aligned}
& \text { Health Performance for a building }=\sum_{\mathrm{i}=1}^{1} \mathrm{HP}_{\mathrm{SPACEi}} \\
& =\sum^{1} \sum^{\mathrm{m}} \mathrm{W}_{\mathrm{HFj}} \mathrm{HP}_{\mathrm{HFj}}=\sum^{1} \sum^{\mathrm{m}} \mathrm{W}_{\mathrm{HFj}} \times \sum^{\mathrm{n}} \mathrm{W}_{\mathrm{EFk}} \mathrm{HP}_{\mathrm{EFk}}
\end{aligned}
$$

Where, the terms appearing in Eq. (1) are defined as follows

HP $_{\text {SPACEi }}$ : Health performance of each space

i: Number of spaces $(1, \ldots, 1)$

$\mathrm{W}_{\mathrm{HFj}}$ : Weight of each health factor

$\mathrm{HP}_{\mathrm{HFj}}$ : Health performance of each health factor

$\mathrm{j}$ : Number of factors $(1, \ldots, \mathrm{m})$

$\mathrm{W}_{\mathrm{EFk}}$ : Weight of each evaluation factor

$\mathrm{HP}_{\mathrm{EFk}}$ : Health performance of each evaluation factor $\mathrm{k}$ : Number of spaces $(1, \ldots, \mathrm{n})$.

$\mathrm{HP}_{\text {design }} \mathrm{HP}_{\text {require ? }}$

Where, the terms appearing in Eq. (2) are defined as follows

$\mathrm{HP}_{\text {design }}$ : Health performance in the design phase

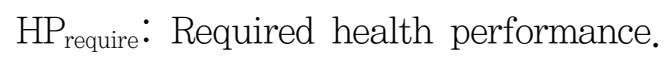

When the health performance in the design phase meets functional requirements in the planning phase, construction of the building begins. Otherwise, feedback is offered to engage in a redesign process to enhance health performance. Resources are selected on the basis of HRDB for construction. When the health performance measured in the construction phase meets that of the design phase, construction is completed and the maintenance phase is initiated. If the health performance falls below the baseline in the course of the construction phase, resources shall be modified to adjust the performance level. Finally, when requirements are met in the operation and management phase, a sustainable healthy building is ensured. Otherwise, the operation and management plan needs to be revised to adjust the health performance level. 


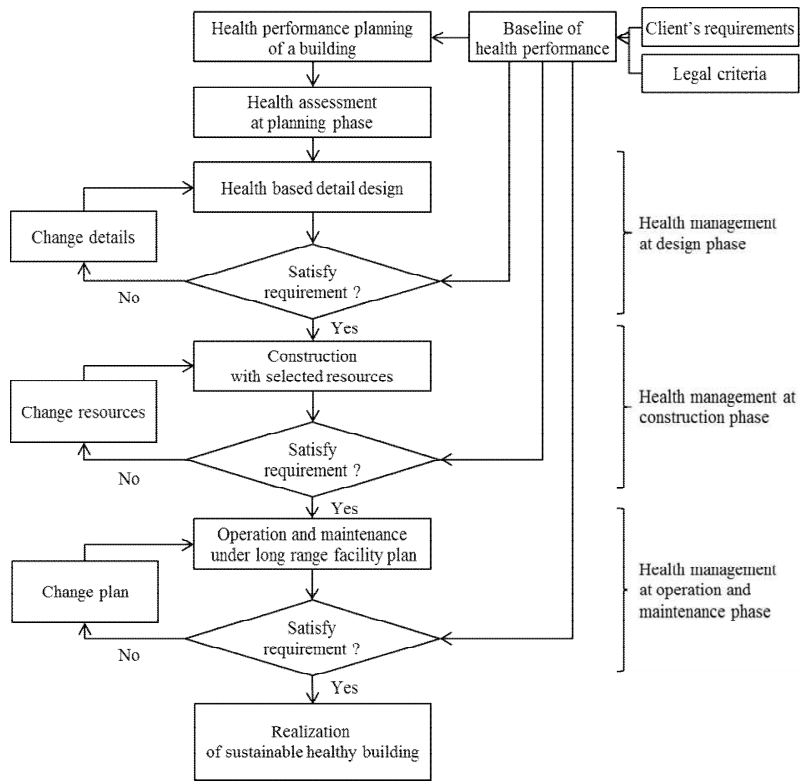

Figure 9. Health management flow throughout the building lifecycle

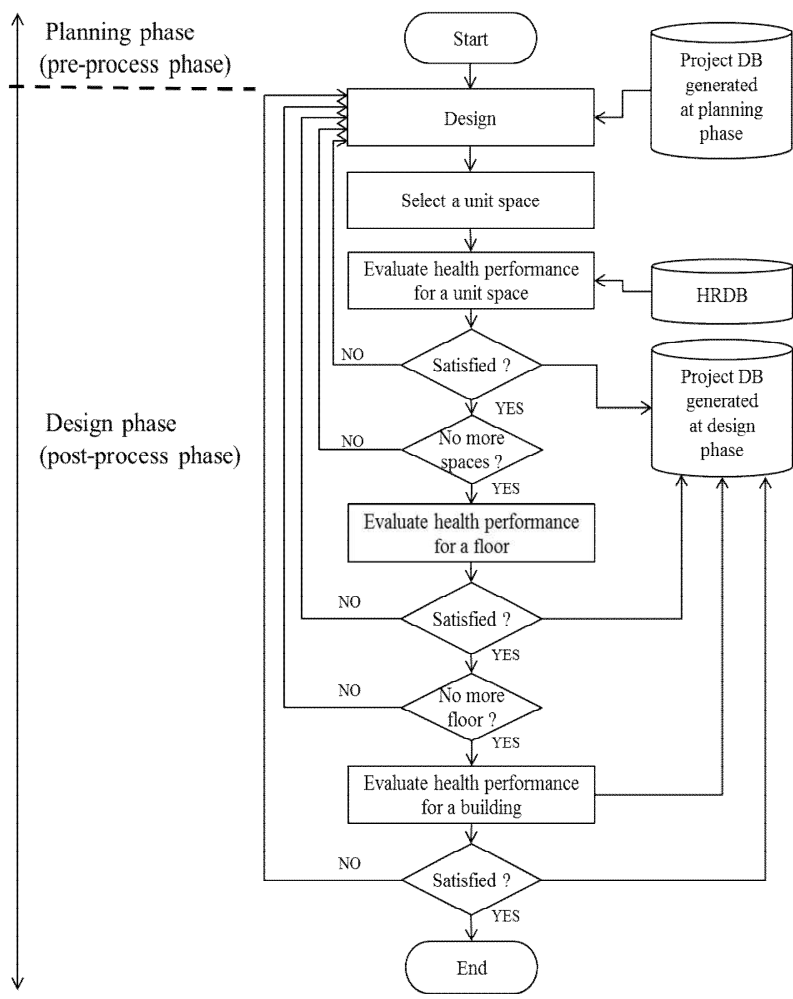

Figure 10. Detailed process of health assessment in the design phase

Figure 10 shows the details of such procedures in the design phase. First, the building is designed to satisfy the legal criteria, client requirements, and planner's decisions as specified in the project DB produced during the planning phase. Second, spaces such as living rooms, bedrooms, and kitchens on specific floors are selected using the LHT model. Third, the health performance of resources is measured using HRDB by area. Fourth, the health performance of a unit space is examined to determine whether it meets requirements. When it fails to meet requirements, the space is redesigned using feedback to satisfy corresponding requirements (LHM). Fifth, it is necessary to examine whether all spaces on corresponding floors have undergone the health performance assessment. Sixth, health performance assessments of all spaces are totaled up upon completion of the health performance assessment. Seventh, it is necessary to examine whether the health performance of corresponding floors satisfies requirements. Eighth, it is necessary to examine whether all floors underwent the health performance assessment. The health performance of the following floors is then measured. Ninth, the health performance assessments of different floors are totaled up to evaluate the comprehensive building health performance. Lastly, the design phase is complete when the entire building's health performance meets requirements. The health performance data measured in each space, floor, and building unit is stored in the project $\mathrm{DB}$ of the planning phase and redirected to the database for the construction phase. However, only the health performance of floors and building units used for the main purposes of the building are totaled up in this process.

Organizing the specific evaluation process of health performance in the design phase, building health performance is assessed on the basis of data output and planned from the planning phase (a pre-process phase). The LHM module must maintain the health performance level measured in the post-process phase above the baseline of health performance[11]. 


\subsection{Database relation of the LHA model}

Figure 11 shows the database relation of the LHA model. By using LHT module and HPE module, building space and SCIS can be defined. According to this data, health performance of a building can be verified.

A sustainable healthy building needs to be designed in consideration of a detailed description of the building and the HRDB, and to be defined with spaces and SCIs. The outcome is stored in the space DB and SCI DB. These data are built up into the hierarchical structure and saved into the LHT DB. The LHT DB data is then retrieved into EFs and HFs in the indicator database (Ind DB). After evaluating the health performance of spaces and resources with the information retrieved from Space DB, SCI DB, and Ind $\mathrm{DB}$, the results are saved into HPE DB.

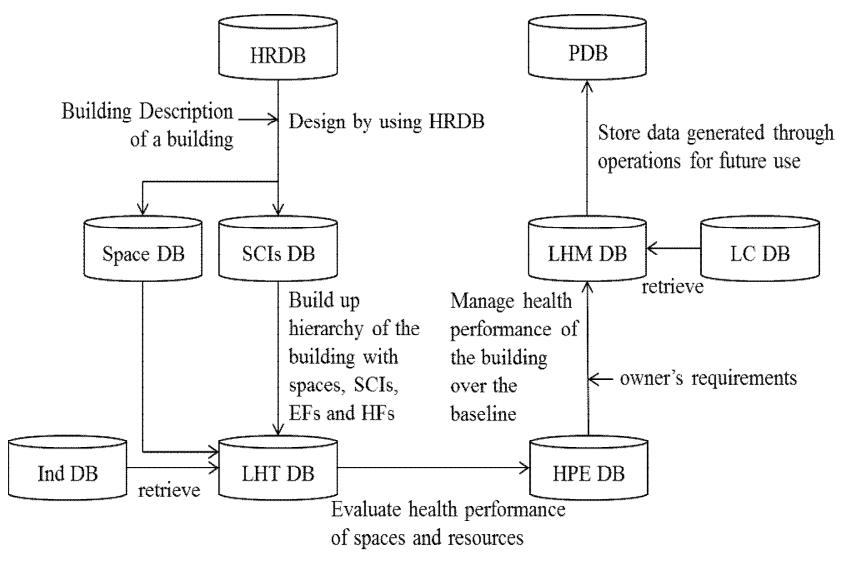

Figure 11. DB relation of LHA model

The health performance data evaluated in the previous process are then managed over the baseline, which includes the owner's requirements and legal criteria (LC DB), and the pertinent information from the LHM process is saved into the LHM DB. If the health performance retrieved from HPE DB fails to satisfy the baseline, the health performance is repetitively evaluated after spaces or SCIs are corrected until the baseline is satisfied as described in Figure 10. The health performance adjusted using LHM is saved into PDB along with the project outline and LHM history for use in following projects. HRDB is categorized as general data applied to all projects, while the remaining data is categorized as project data produced from corresponding projects.

The entity-relation diagram between DBs in Figure 11 is constructed as shown in Figure 12. For instance, data stored in the LHM DB such as the health performance baseline for management (HP_HASELINE), lifecycle phase of building (LC_PHASE), health performance target for evaluation (HP_TARGET), equations for re-evaluation (HP_EQUATION), original value of health performance (HP_VALUEORIGN), and managed value of health performance (HP_VALUEMODIFY) are managed using LHM code (LHM_CODE), a primary key (PK). They contain project code (PROJ_CODE), a foreign key (FK) for links with other DBs.

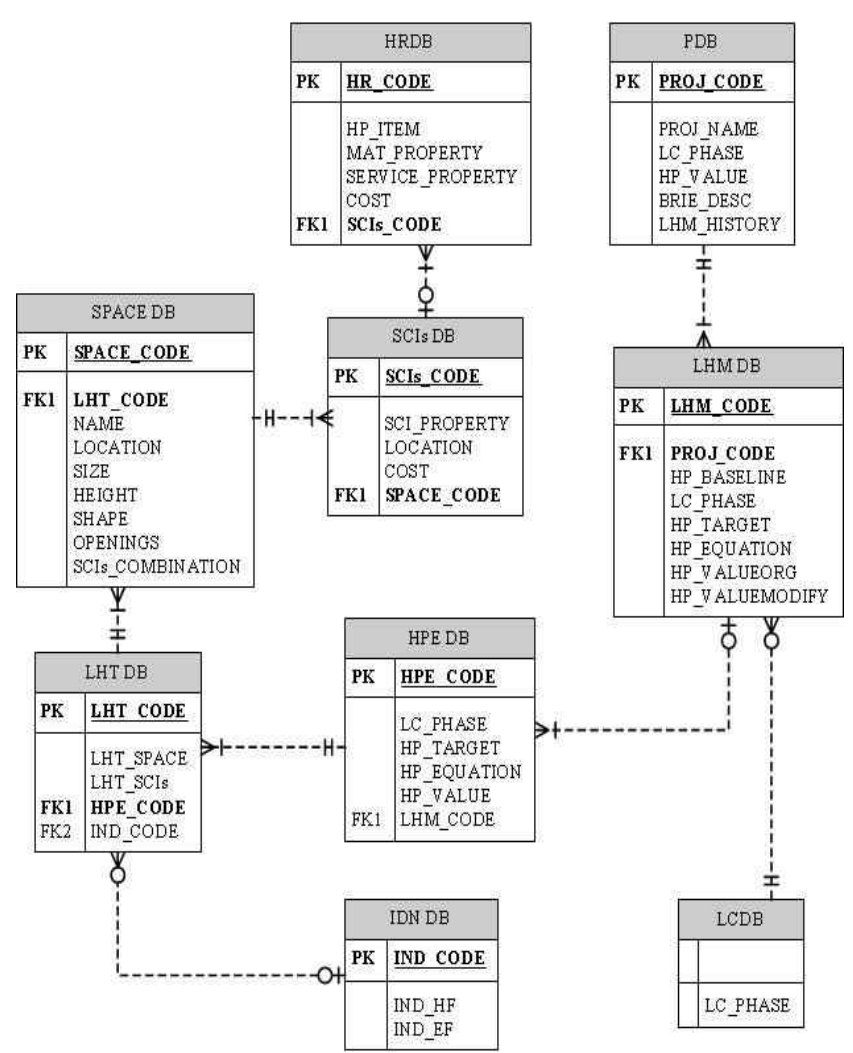

Figure 12. E-R diagram of the LHA model 


\section{Discussion and conclusion}

Previous studies of health performance over the building lifecycle have focused on assessing and evaluating the health performance of individual factors (light, heat, etc.). However, comprehensive management in consideration of the health performance for residents (from planning to demolition) has not been sufficiently studied. Since financial investments and efforts are required to enhance a building's health performance, it must be consistently managed and improved in an appropriate manner. Therefore, this study developed the lifecycle health assessment model for sustainable buildings, which led to the following outcomes.

First, the concepts of LHA are suggested in association with the concepts of HRDB, LHT, and HPE to calculate the health performance of buildings in accordance with their lifecycles. LHA associated with those modules offers the structure (LHT), information (HRDB), and methodologies (HPE) to assess the health performance of a building throughout its lifecycle quickly and easily. The outcomes resulting from LHA can also be shared.

Second, the concepts of lifecycle health management (LHM) are suggested. LHM is designed to maintain the health performance (measured using HPE by the lifecycle) above the baseline in order to ensure sustainable healthy buildings.

Third, the operating concepts of the LHA model to be applied for the building are presented while the application procedure is specified. The design phase is described in detail as an example in this study. This example is also applicable to the planning, construction, and operation and management phases.

Fourth, the DB relation and entity-relation diagrams are suggested to clarify the information relations that operate within the LHA model. It is neces- sary to develop the computing system, which can be established easily and rapidly when the properties and values of data are clarified.

The LHA model presented in this study includes concepts to manage health performance according to legal and client requirements, within the project budget. This implies that required building performance can be achieved with minimum cost. For further study, establishing a system that clarifies EFs and HFs will expedite the assessment of health performance based on a variety of design details.

\section{Acknowledgements}

This work was supported by a National Research Foundation of Korea (NRF) grant funded by the Korea government (MSIP) (No. 2008-0061908).

\section{References}

1. Jeong EY. A concept and evaluation system of building design performance. Journal of the Architectural Institute of Korea. 2000 Jul;44(7):23-27.

2. Loe HW. The healthy house. Review of Architecture and Building Science. 2005 May;49(5):34-36.

3. Lee GJ, Na YJ, Kim JT, Kim SK. A computing model for lifecycle health performance evaluations of sustainable healthy buildings. Indoor and Built Environment. 2013 Feb;22(1):7-20.

4. Cho SH, Choi IY, Jung SJ. Structure of indicators evaluating healthy housing quality in apartments. Proceeding of the 2010 spring annual conference of the Korea institute of ecological architecture and environment; 2010 May; Seoul, Korea. Seoul (Korea): Korea institute of ecological architecture and environment; 2010. p. 149-54.

5. Yu CWF, Kim JT. Building environmental assessment schemes for rating of IAQ in sustainable buildings. Indoor Built Environment 2011 Jan;20(1):5-15.

6. Buratti C, Mariani R, Moretti E. Mean age of air in a naturally ventilated office, Experimental data and simulations. Energy and Buildings. 2011 Aug;43:2021-7.

7. Yu CWF, Kim JT. Building pathology investigation of sick buildings - VOC emissions. Indoor and Built Environment. 2010 
Feb;19(1):30-9.

8. Lee SH, Kwon GD, Joo JK, Kim JT, Kim SK. A finish material management system for indoor air quality of apartment buildings (FinIAQ). Energy and Buildings. 2012 Mar;46:68-79.

9. Lee DH, Lee SH, Kim JT, Kim SK. A lifecycle health performance tree for sustainable healthy buildings. Indoor and Built Environment. 2012 Jan;21(1):16-27.

10. Zheng Q, Loe DH, Loe SH, Kim JT, Kim SK. A health performance evaluation model of apartment building indoor air quality. Indoor and Built Environment. 2011 May; 20(1):26-35.

11. Lim CY, Joo JK, Kim JT, Kim SK. Lifecycle health assessment model of apartment Buildings. Proceeding of International Joint Symposium on Renewable Energy Sources and Healthy Buildings; 2013 Apr 21-3; Belgrade, Serbia. Belgrade (Serbia): Serbian Society for Heating, Refrigeration and Air Conditioning; 2013. p. 22-3.

12. Wikipedia. Indoor air quality [Internet]. Wikimedia Foundation, Inc.; 2013 Jul [cited 2013 Aug]. Available from: http://en.wikipedia.org/wiki/Indoor_air_quality

13. Zheng Q, Lee DH, Choi JH, Kim SK. A health performance evaluation model of building indoor air quality. Journal of the Korea Institute of Ecological Architecture and Environment. 2010 Jun;10(3):3-10.

14. Kwon GD, Kim SK. A study on a database management system for health-friendly building materials. Journal of the Korea Institute of Ecological Architecture and Environment. 2009 Dec; 9(6):3-9. 